\title{
CS Research Square \\ Predictors of Postnatal Depression in the Slums Nairobi, Kenya: A Cross-Sectional Study
}

\section{University of Nairobi \\ Mary Wangari Kuria \\ University of Nairobi \\ Fredrick Namenya Were \\ University of Nairobi \\ David Musyimi Ndetei \\ University of Nairobi}

Esther Wanjiku Kariuki ( $\nabla$ kariukiesther@yahoo.com )

\section{Research Article}

Keywords: Postnatal depression, predictors, $\mathrm{MCH}$, low-resource, slums

Posted Date: July 9th, 2021

DOl: https://doi.org/10.21203/rs.3.rs-688703/v1

License: (c) (1) This work is licensed under a Creative Commons Attribution 4.0 International License. Read Full License

Version of Record: A version of this preprint was published at BMC Psychiatry on April 5th, 2022. See the published version at https://doi.org/10.1186/s12888-022-03885-4. 


\section{Abstract}

\section{Background}

Postnatal depression (PND) is a universal mental health problem that prevents mothers' optimal existence and mothering. Although research has shown high PND prevalence rates in Africa, including Kenya, little research has been conducted to determine the contributing factors, especially in low-resource communities.

\section{Objective}

This study aimed to investigate the PND risk factors among mothers attending Maternal and Child Health Clinics (MCH) in the slums, Nairobi.

\section{Methods}

This study is cross-sectional, which is a part of a larger study. A sample of 567 mothers of 6-10 weeks postnatal from two Maternal and Child Health $(\mathrm{MCH})$ formed the study population. The Depression rate was measured using the original 1961 Beck's Depression Inventory (BDI). In addition, a sociodemographic questionnaire (SDQ) was used to collect hypothesized risk variables.

\section{Results}

The PND prevalence rate was $27.1 \%$. Women with: unplanned pregnancy ( $\mathrm{AOR}=1.87,95 \% \mathrm{Cl} 1.02,3.43)$, unemployed $(A O R=4.43,95 \% \mathrm{Cl} 1.01,19.76)$, dissatisfied with body image ( $A O R=2.51,95 \% \mathrm{Cl} 1.21,5.19)$ and feeling fatigued (AOR=2.02,95\% $\mathrm{Cl} 1.06,3.85)$ had higher odds of developing PND.

\section{Conclusion}

This study builds upon scarce previous studies on PND from low-income countries. Identifying specific PND risk factors may help in devising targeted prophylactic and therapeutic strategies.

\section{Background}

Postnatal depression (PND) is a universal mental health problem(1) across countries and cultures(2). The depression prevalence rates are higher (5.2\% and $74.0 \%$ ) in developing countries compared to $1.9 \%$ to $82.1 \%$ in the developed countries(2). In Africa, a systematic review and meta-analysis reported an overall pooled PND prevalence of $16.84 \%$. Published research studies conducted in Kenya report high PND prevalence rates(3), (4), (5) in different settings.

Symptoms of PND include depressed mood, anxiety, anhedonia(6), fatigue(7), sleep difficulties(8) and concentration problems(8). As a consequence, mothers parenting capacities get diminished(9), which in turn leads to childs' poor: - physical health(10), growth(11), and poor motherchild relationship(12). As a consequence, leading to poor childs' developmental outcomes(13). 
Postnatal depression is caused by many factors: - psychosocial, socioeconomic, obstetric, and hormonal variables(14). Some of the main psychosocial risk factors include low: - maternal age(15), income(16), and education(17). Mothers experiencing stressful life events(18), lack of social support(19), poor marital relationship(20) and domestic violence(21) are also at risk for PND. Besides, infant characteristics like adverse birth and infant health outcomes(22), difficult temperament(23) and unwanted gender(24) are risk factors. Mothers with poor physical health (25), poor obstetric histories(26), unplanned pregnancy(27), and a history of psychiatric illness (28) are likely to have PND. Moreover, poor environmental conditions(29) and cultural practices(30), (31) are also PND predictors.

Although PND is a public health problem, African countries(32), including Kenya, have neglected it. Therefore, more research is needed due to the scarcity of published work in this area(22). Moreover, it is crucial to investigate and understand PND risk factors to devise targeted prevention and treatment strategies(33). This study aimed to investigate PND predictors in early postpartum period (6-10 weeks) in two low-resourced urban communities.

\section{Methods}

\section{Participants and Procedures}

This paper is a cross-sectional design and is a part of a longitudinal study (34) and investigated hypothesized risk factors for PND. Data was collected from Lang'ata and Riruta Health Centres- $\mathrm{MCH}$ clinics. Both are situated in Nairobi county and serve low-resource communities.

Mothers were recruited as they brought their infants for the first $\mathrm{MCH}$ clinic visit through continuous purposive sampling until the required sample was achieved(567 participants). The sampling method is published elsewhere (34). Informed consenting mothers filled up a self-administered Social-Demographic Questionnaire (SDQ) and an English version of the original (1961) Becks Depression Inventory (BDI).

We obtained ethical approval from Kenyatta National Hospital Ethical Committee, Office of the President through the Ministry of Higher Education Science and Technology and Medical Officer of Health (MOH), Nairobi County.

\section{Data collection instruments}

\section{Sociodemographic questionnaire}

A sociodemographic questionnaire to collect personal information and hypothesized PND risk factors that include: - mothers' age in years; educational level; marital status; monthly household income; suffering from chronic illness; satisfaction with body image; conflict with any close relatives; have a stressful life event; pregnancy planned; happy with infants' health; not able to work (fatigue) and age of the infant and gestational age. 


\section{Beck's Depression Inventory (BDI)}

The original Beck's Depression Inventory (BDI), first published in 1961, was created by Dr Aaron T. Beck (Beck et al., 1961) and later revised in 1971. Beck's Depression Inventory is a self-administered report which takes approximately 10 minutes to complete with demonstrated consistent properties over time. It has a good Internal consistency, with a Cronbach's alpha coefficient of around 0.85 (Ambrosini et al. 1991). It is also positively correlated with the Hamilton Depression Scale (Brown et al., 1995) with a Pearson ratio of 0.71. The test was also found to have a high one-week test-retest with a Pearson value of 0.93 (Beck, Steer and Brown, 1996). A total score is calculated as the sum of the 21 items with a range of $0-63$. The clinical cut-offs are 11-16 (mild mood disturbance), 17-20 (borderline clinical depression), 21-30 (moderate depression), 31-40 (severe depression), and 40-63 (extreme depression). The BDI has been used in Kenya and other countries (35-38). BDI is a self-administered report which takes approximately 10 minutes to complete.

\section{Data analysis}

Item means, standard deviations, frequencies and percentages were calculated for the sociodemographic, psychosocial related variables. The association between each independent variable and the dependent variable was assessed in bivariate analyses. Those independent variables with P-value $<0.25$ were entered into a multivariate logistic regression to control the cofounders and identify PND predictors using the enter method. P-value of $<0.05$ was used as the criterion for statistical significance, and an OR with a $95 \%$ confidence interval was used to indicate the strength of association. All analysis was conducted with IBM SPPS v 23.

\section{Results}

Of the total 591 eligible mothers, 575 participated in the study, which made a response rate of $97.3 \%$.

\section{Sociodemographics and other characteristics of the participants}

Table 1 reports the characteristics of the participants in our sample. The mean age was 25.9 years and ranged from $18-40$. The majority of the participants (45.1\%) were aged between $18-24$ years; $37.6 \%$ were aged between $25-30$ years; $17.3 \%$ were aged between $30-40$ years. A majority (89.8\%) were married, more than half $(53.4 \%)$ had a secondary level of education, nearly $36.5 \%$ had a primary education level, the rest, $10.1 \%$, had a tertiary level of education. A majority $(67.7 \%)$ of the participants were earning income below 20,000 (196\$) per month. $5.1 \%$ of the women had been suffering from chronic illness, $86.9 \%$ were satisfied with their body, $11.6 \%$ had a conflict with their relatives, $28.4 \%$ had a stressful life event, $67.4 \%$ had planned their pregnancy, $66.7 \%$ were happy with baby's health, and $23.8 \%$ had work-related problems (fatigue). A majority $(70.5 \%)$ had infants aged $7-10$ weeks, while the rest had infants aged 6 weeks. About $9.3 \%$ had babies born before 37 weeks (Pre-term births). 
Table 1

Characteristics of the Respondents

\begin{tabular}{|c|c|c|c|}
\hline Variable & Category & Frequency $(N=567)$ & Percentage (\%) \\
\hline \multirow[t]{3}{*}{ Age in Years } & $18-24$ Years & 256 & 45.1 \\
\hline & $25-30$ Years & 213 & 37.6 \\
\hline & $31-40$ & 98 & 17.3 \\
\hline Age years & Mean, Range & 25.9 & $18-40$ \\
\hline \multirow[t]{3}{*}{ Education Level } & Primary & 207 & 36.5 \\
\hline & Secondary & 303 & 53.4 \\
\hline & Graduate and above & 57 & 10.1 \\
\hline \multirow[t]{2}{*}{ Marital Status } & Currently married & 509 & 89.8 \\
\hline & Currently not Married & 58 & 10.2 \\
\hline \multirow[t]{3}{*}{ Employment Status } & Employed & 85 & 15 \\
\hline & Self-employed & 102 & 18 \\
\hline & Unemployed & 380 & 67 \\
\hline \multirow[t]{4}{*}{ Monthly Income } & $<10,000$ & 198 & 34.9 \\
\hline & $10,000-19,000$ & 186 & 32.8 \\
\hline & $20,000-29,000$ & 103 & 18.2 \\
\hline & 30,000 and Above & 80 & 14.1 \\
\hline \multirow[t]{2}{*}{ Suffering from Chronic Illness } & No & 538 & 94.9 \\
\hline & Yes & 29 & 5.1 \\
\hline \multirow[t]{2}{*}{ Satisfied with body image } & No & 74 & 13.1 \\
\hline & Yes & 493 & 86.9 \\
\hline \multirow[t]{2}{*}{ Conflict with any close relatives } & No & 501 & 88.4 \\
\hline & Yes & 66 & 11.6 \\
\hline \multirow[t]{2}{*}{ Have a Stressful life Event } & No & 406 & 71.6 \\
\hline & Yes & 161 & 28.4 \\
\hline \multirow[t]{2}{*}{ Pregnancy Planned } & No & 185 & 32.6 \\
\hline & Yes & 382 & 67.4 \\
\hline Happy with baby's Health & No & 191 & 33.7 \\
\hline
\end{tabular}




\begin{tabular}{|llll|}
\hline Variable & Category & Frequency $(\mathbf{N}=567)$ & Percentage (\%) \\
\hline \multirow{2}{*}{ Have Work Problems(fatigue) } & Yes & 376 & 66.3 \\
\cline { 2 - 4 } & No & 432 & 76.2 \\
\cline { 2 - 4 } Age of the infant & Yes & 135 & 23.8 \\
\cline { 1 - 3 } & 7-10 Weeks & 167 & 70.5 \\
\hline Gestation at birth & $<37$ Weeks & 53 & 29.5 \\
\cline { 2 - 4 } & $>=37$ Weeks & 514 & 90.3 \\
\hline
\end{tabular}

\section{Prevalence of Postpartum depression}

Based on the original (1961) Becks Depression Index (BDI0 cut-off points, the scores as follows, Normal (0$10),(n=414,73.0 \%)$; Mild mood disturbance (11-16); $(n=65,11.5 \%)$; Borderline clinical depression (17$20) ;(n=30,5.3 \%)$; Moderate depression (21-30); $(n=42,7.4 \%)$; Severe depression (31-40); $(n=10,1.8 \%)$ and Extreme depression $(40+) ;(n=6,1.1 \%)$. Therefore, the PND prevance is $27.0 \%$. The mean BDI scores was $7.7, \mathrm{SD}=8.9$ and ranged from $0-54$.

Table 2

Prevalence of Depression among the respondents

\begin{tabular}{|c|c|c|c|}
\hline Variable & Category & $\begin{array}{l}\text { Frequency } \\
(\mathrm{N}=567)\end{array}$ & $\begin{array}{l}\text { Percentage } \\
\text { (\%) }\end{array}$ \\
\hline \multirow{6}{*}{$\begin{array}{l}\text { Depression Levels (Beck Depression } \\
\text { Inventory-BDI) }\end{array}$} & Normal $(0-10)$ & 414 & 73 \\
\hline & $\begin{array}{l}\text { Mild mood disturbance (11- } \\
\text { 16) }\end{array}$ & 65 & 11.5 \\
\hline & $\begin{array}{l}\text { Borderline Clinical Depression } \\
(17-20)\end{array}$ & 30 & 5.3 \\
\hline & Moderate Depression $(21-30)$ & 42 & 7.4 \\
\hline & Severe Depression $(31-40)$ & 10 & 1.8 \\
\hline & Extreme depression (40+) & 6 & 1.1 \\
\hline \multirow[t]{2}{*}{ Depression status } & Normal & 509 & 89.8 \\
\hline & Elevated & 58 & 27.1 \\
\hline Depression (Scores) & Mean \pm SD & 7.73 & 8.91 \\
\hline
\end{tabular}

\section{Factors associated with postnatal depression}

Multivariate logistic regressions revealed; those who had an unplanned pregnancy were 1.87 times more likely to develop PND as compared to planned pregnancy ( $\mathrm{AOR}=1.87,95 \% \mathrm{Cl} 1.02,3.43$ ), those who were 
unemployed were 4.43 times more like to develop PND as compared to those who were employed (AOR = $4.43,95 \% \mathrm{Cl} 1.01,19.76)$. Participants who were unsatisfied with their body image were 2.51 times more likely to develop PND as compared to those who were satisfied with their body image (AOR $=2.51,95 \% \mathrm{Cl}$ $1.21,5.19$ ). Participants who had work-related problems (fatigue) were 2.02 times more likely to develop PND than those without work-related problems $(A O R=2.02,95 \% \mathrm{Cl} 1.06,3.85)$. 
Table 3

Factors associated with postpartum depression

\begin{tabular}{|c|c|c|c|c|c|c|c|}
\hline \multirow[t]{2}{*}{ Variable } & \multirow[t]{2}{*}{ Category } & \multicolumn{2}{|c|}{ Post-Partum Depression } & \multirow{2}{*}{$\begin{array}{l}\text { O.R. }(95 \% \\
\text { C.I) }\end{array}$} & \multirow{2}{*}{$\begin{array}{l}\mathrm{p}- \\
\text { value }\end{array}$} & \multirow{2}{*}{$\begin{array}{l}\text { aO.R. }(95 \% \\
\text { C.I) }\end{array}$} & \multirow{2}{*}{$\begin{array}{l}\mathrm{p} \text { - } \\
\text { value }\end{array}$} \\
\hline & & No & Yes & & & & \\
\hline \multirow[t]{3}{*}{ Age in Years } & $\begin{array}{l}18-24 \\
\text { Years }\end{array}$ & $221(86.3 \%)$ & $35(13.7 \%)$ & $\begin{array}{l}2.06(0.88- \\
4.80)\end{array}$ & 0.095 & $\begin{array}{l}1.78(0.71- \\
4.48)\end{array}$ & 0.222 \\
\hline & $\begin{array}{l}25-30 \\
\text { Years }\end{array}$ & 197(92.5\%) & $16(7.5 \%)$ & $\begin{array}{l}1.06(0.42- \\
2.66)\end{array}$ & 0.908 & $\begin{array}{l}1.36(0.50- \\
3.68)\end{array}$ & 0.549 \\
\hline & $31-40$ & $91(92.9 \%)$ & $7(7.1 \%)$ & Ref. & & Ref. & \\
\hline \multirow[t]{3}{*}{$\begin{array}{l}\text { Education } \\
\text { Level }\end{array}$} & Primary & $171(82.6 \%)$ & $36(17.4 \%)$ & $\begin{array}{l}3.79(1.12- \\
12.80)\end{array}$ & 0.032 & $\begin{array}{l}1.82(0.49- \\
6.79)\end{array}$ & 0.370 \\
\hline & Secondary & 284(93.7\%) & $19(6.3 \%)$ & $\begin{array}{l}1.20(0.34- \\
4.21)\end{array}$ & 0.771 & $\begin{array}{l}0.75(0.20- \\
2.85)\end{array}$ & 0.678 \\
\hline & $\begin{array}{l}\text { Graduate } \\
\text { and above }\end{array}$ & $54(94.7 \%)$ & $3(5.3 \%)$ & Ref. & & Ref. & \\
\hline \multirow[t]{2}{*}{$\begin{array}{l}\text { Marital } \\
\text { Status }\end{array}$} & $\begin{array}{l}\text { Currently } \\
\text { married }\end{array}$ & $460(90.4 \%)$ & $49(9.6 \%)$ & Ref. & & Ref. & \\
\hline & $\begin{array}{l}\text { Currently } \\
\text { not Married }\end{array}$ & $49(84.5 \%)$ & $9(15.5 \%)$ & $\begin{array}{l}1.72(0.80- \\
3.72)\end{array}$ & 0.165 & $\begin{array}{l}1.54(0.63- \\
3.80)\end{array}$ & 0.344 \\
\hline \multirow{3}{*}{$\begin{array}{l}\text { Employment } \\
\text { Status }\end{array}$} & Employed & $83(97.6 \%)$ & $2(2.4 \%)$ & Ref. & & Ref. & \\
\hline & $\begin{array}{l}\text { Self- } \\
\text { employed }\end{array}$ & $98(96.1 \%)$ & $4(3.9 \%)$ & $\begin{array}{l}1.69(0.30- \\
9.48)\end{array}$ & 0.549 & $\begin{array}{l}1.58(0.27- \\
9.31)\end{array}$ & 0.615 \\
\hline & Unemployed & $328(86.3 \%)$ & $52(13.7 \%)$ & $\begin{array}{l}6.58(1.57- \\
27.57)\end{array}$ & 0.010 & $\begin{array}{l}4.43(1.01- \\
19.76)\end{array}$ & 0.050 \\
\hline \multirow[t]{4}{*}{$\begin{array}{l}\text { Monthly } \\
\text { Income }\end{array}$} & $<10,000$ & $169(85.4 \%)$ & $29(14.6 \%)$ & $\begin{array}{l}4.40(1.30- \\
14.90)\end{array}$ & 0.017 & $\begin{array}{l}1.97(0.52- \\
7.51)\end{array}$ & 0.318 \\
\hline & $\begin{array}{l}10,000- \\
19,000\end{array}$ & $166(89.2 \%)$ & $20(10.8 \%)$ & $\begin{array}{l}3.09(0.89- \\
10.72)\end{array}$ & 0.075 & $\begin{array}{l}2.02(0.53- \\
7.63)\end{array}$ & 0.302 \\
\hline & $\begin{array}{l}20,000- \\
29,000\end{array}$ & $97(94.2 \%)$ & $6(5.8 \%)$ & $\begin{array}{l}1.59(0.38- \\
6.55)\end{array}$ & 0.523 & $\begin{array}{l}1.24(0.28- \\
5.55)\end{array}$ & 0.781 \\
\hline & $\begin{array}{l}30,000 \text { and } \\
\text { Above }\end{array}$ & 77(96.3\%) & $3(3.8 \%)$ & Ref. & & Ref. & \\
\hline Suffering & No & $485(90.1 \%)$ & $53(9.9 \%)$ & Ref. & & Ref. & \\
\hline $\begin{array}{l}\text { Chronic } \\
\text { Illness }\end{array}$ & Yes & $24(82.8 \%)$ & $5(17.2 \%)$ & $\begin{array}{l}1.91(0.70- \\
5.20)\end{array}$ & 0.208 & $\begin{array}{l}1.57(0.49- \\
5.07)\end{array}$ & 0.448 \\
\hline \multirow{2}{*}{$\begin{array}{l}\text { Satisfied } \\
\text { with body } \\
\text { image }\end{array}$} & No & $60(81.1 \%)$ & $14(18.9 \%)$ & $\begin{array}{l}2.38(1.23- \\
4.60)\end{array}$ & 0.010 & $\begin{array}{l}2.51(1.21- \\
5.19)\end{array}$ & 0.013 \\
\hline & Yes & $449(91.1 \%)$ & $44(8.9 \%)$ & Ref. & & Ref. & \\
\hline
\end{tabular}




\begin{tabular}{|c|c|c|c|c|c|c|c|}
\hline \multirow{2}{*}{$\begin{array}{l}\text { Have a } \\
\text { Stressful life } \\
\text { Event }\end{array}$} & No & $373(91.9 \%)$ & $33(8.1 \%)$ & Ref. & & & \\
\hline & Yes & $136(84.5 \%)$ & $25(15.5 \%)$ & $\begin{array}{l}2.08(1.19- \\
3.62)\end{array}$ & 0.010 & $\begin{array}{l}1.70(0.92- \\
3.13)\end{array}$ & 0.090 \\
\hline \multirow[t]{2}{*}{$\begin{array}{l}\text { Pregnancy } \\
\text { Planned }\end{array}$} & No & $157(84.9 \%)$ & $28(15.1 \%)$ & $\begin{array}{l}2.09(1.21- \\
3.62)\end{array}$ & 0.008 & $\begin{array}{l}1.87(1.02- \\
3.43)\end{array}$ & 0.043 \\
\hline & Yes & $352(92.1 \%)$ & $30(7.9 \%)$ & Ref. & & & \\
\hline \multirow{2}{*}{$\begin{array}{l}\text { Have Work } \\
\text { Problems } \\
\text { (fatigued) }\end{array}$} & No & $395(91.4 \%)$ & $37(8.6 \%)$ & Ref. & & Ref. & \\
\hline & Yes & $114(84.4 \%)$ & $21(15.6 \%)$ & $\begin{array}{l}1.97(1.11- \\
3.49)\end{array}$ & 0.021 & $\begin{array}{l}2.02(1.06- \\
3.85)\end{array}$ & 0.032 \\
\hline \multirow[t]{2}{*}{$\begin{array}{l}\text { Age of the } \\
\text { infant }\end{array}$} & 6 Weeks & $360(90.0 \%)$ & $40(10.0 \%)$ & $\begin{array}{l}0.92(0.51- \\
1.66)\end{array}$ & 0.780 & $\mathrm{n} / \mathrm{s}$ & \\
\hline & 7-10 Weeks & $149(89.2 \%)$ & $18(10.8 \%)$ & Ref. & & & \\
\hline
\end{tabular}

\section{Discussion}

The prevalence of PND was 27.1\%. Published literature from Kenya shows high PND prevalence rates(3), (4), (5) in different settings. Research findings from other African countries also show a high PND prevalence rate: Rwanda 63.6\%, South Africa (57.14\%) (39) and (38.8\%) (40), Nigeria 35.6\% (19). Comparable to our finding, a systematic review and meta-analysis revealed a prevalence rate of $26 \%$ in Middle-East countries, while European countries had lower rates (8\%) (41).

This study confirms that unplanned pregnancy is a risk factor for PND (42). Probably the unwanted pregnancy stressed the mothers due to the circumstances surrounding the pregnancy. For example, unplanned pregnancy has negative consequences that include; stigma, perceived loss of opportunities(43), poor health(44) and unhappiness(45).

Unemployed women had higher PND symptoms than the employed in this study, as other studies show(46), (47). A possible explanation is that unemployment could have exposed women to financial stress. A difficult financial situation may contribute to PND development(48). Besides, unemployed women are at risk of domestic abuse(49), which in turn may cause PND development(50).

Women who felt fatigued and unable to perform the usual household chores after giving birth were more depressed than those who did not. In a study correlating with our findings, Giallo et al. found that mothers in the high-risk depressive symptoms group were most likely to complain of fatigue (51). However, postnatal fatigue should be best understood as separate psychological constructs or experiences(52).

\section{Limitations}

The study participants resided in urban slums and therefore did not represent the rest of the city population. This study was a cross-sectional design and could not determine cause and effect. 


\section{Conclusion}

This study builds upon scarce previous studies on PND from low-income countries. Identifying specific PND risk factors may help in devising targeted prophylactic and therapeutic strategies. More research is needed to determine the role of cultural factors in PND development.

\section{Abbreviations}

PND: Postnatal Depression

$\mathrm{MCH}$ : Maternal and Child Health

SDQ: Sociodemographic Questionnaire

BDI: Beck's Depression Inventory

MOH: Medical Officer of Health

\section{Declarations}

\section{Ethics approval and consent to participate:}

Approval was obtained from Kenyatta National Hospital Ethical Committee, Office of the President through the Ministry of Higher Education Science and Technology, the Medical Officer of Health (MOH) Nairobi County. All eligible study participants were explained; the nature and purpose of the study, their rights, procedures, potential risks and benefits of participation before they gave consent.

\section{Consent for publication:}

All authors agree for this article to be published.

\section{Availability of data and material:}

the data supporting the findings of this study are available within the article.

\section{Competing interests:}

The authors declare that they have no competing interests

\section{Funding:}

none 


\section{Authors' contributions:}

This paper is part of a PhD thesis, University of Nairobi. E.W.K. conceived, designed the study and prepared the manuscript. M.W.K supported and gave guidance from the conception and design. F.N.W gave intellectual feedback. D.M.N worked as a senior supervisor and contributed to intellectual feedback. All authors read and approved the final manuscript.

\section{Acknowledgements:}

Not applicable

\section{References}

1. Almond P. Postnatal depression: A global public health perspective. Perspect Public Health. 2009;129(5):221-7.

2. Norhayati MN, Hazlina NN, Asrenee AR, Emilin WW. Magnitude and risk factors for postpartum symptoms: A literature review. J Affect Disord. 2015;175:34-52.

3. Ongeri L, Wanga V, Otieno P, Mbui J, Juma E, Vander Stoep A, Mathai M. Demographic, psychosocial and clinical factors associated with postpartum depression in Kenyan women. BMC Psychiatry. 2018 Oct 1;18(1):1-9.

4. Haithar S, Kuria MW, Sheikh A, Kumar M, Vander Stoep A. Maternal depression and child severe acute malnutrition: a case-control study from Kenya. BMC Pediatr. 2018;18:1-9.

5. Mutua J, Kigamwa P, Tele A, Kumar M. A comparative study of postpartum anxiety and depression in mothers with pre-term births in Kenya. J Affect Disord Reports. 2020;2:100043.

6. Putnam KT, Wilcox M, Robertson-Blackmore E, Sharkey K, Bergink V et al. Clinical phenotypes of perinatal depression and time of symptom onset: analysis of data from an international consortium. The Lancet Psychiatry. 2017;4(6):477-85.

7. Paul S, Corwin EJ. Identifying clusters from multidimensional symptom trajectories in postpartum women. Res Nurs Heal. 2019;42(2):119-27.

8. Santos Jr HP, Kossakowski JJ, Schwartz TA, Beeber L, Fried E. Longitudinal network structure of depression symptoms and self-efficacy in low-income mothers. PLoS One. 2018;13(1):e0191675.

9. Young KS, Parsons CE, Stein A, Kringelbach M. Motion and emotion: depression reduces psychomotor performance and alters affective movements in caregiving interactions. Front Behav Neurosci. 2015;9:26.

10. Scorza P, Owusu-Agyei S, Asampong E, Wainberg M. The expression of perinatal depression in rural Ghana. Int J Cult Ment Health. 2015;8(4):370-81.

11. Nguyen PH, Friedman J, Kak M, Menon P, Alderman $\mathrm{H}$. Maternal depressive symptoms are negatively associated with child growth and development: Evidence from rural India. Matern Child Nutr. 2018;14(4):e12621. 
12. Nakano M, Upadhyaya S, Chudal R, Skokauskas N, Luntamo T, Sourander A et al. Risk factors for impaired maternal bonding when infants are 3 months old: a longitudinal population based study from Japan. BMC Psychiatry. 2019;19(1):1-9.

13. Slomian J, Honvo G, Emonts $P$, Reginster J-Y, Bruyère O. Consequences of maternal postpartum depression: A systematic review of maternal and infant outcomes. Women's Heal. 2019 Apr;15:1745506519844044.

14. Saleh ES, El-Bahei W, del El-Hadidy MA, Zayed A. Predictors of postpartum depression in a sample of Egyptian women. Neuropsychiatr Dis Treat. 2013;9:15.

15. Duko B, Wolde D, Alemayehu Y. The epidemiology of postnatal depression in Ethiopia: a systematic review and meta-analysis. Reprod Health. 2020;17(1):1-9.

16. Niyonsenga J MJ. Factors of postpartum depression among teen mothers in Rwanda: a crosssectional study. J Psychosom Obstet Gynecol. 2020;4:1-5.

17. Decastro F, Hinojosa-Ayala N, Hernandez-Prado B. Risk and protective factors associated with postnatal depression in Mexican adolescents. J Psychosom Obstet Gynecol. 2011;32(4):210-7.

18. Tebeka S, Le Strat Y, Mandelbrot L, Benachi A, Dommergues M, Kayem G et al. Early and late-onset postpartum depression exhibit distinct associated factors: the IGEDEPP prospective cohort study. BJOG An Int J Obstet Gynaecol. 2021.

19. Adeyemo EO, Oluwole EO, Kanma-Okafor OJ, Izuka OM, Odeyemi KA. Prevalence and predictors of postpartum depression among postnatal women in Lagos, Nigeria. Afr Health Sci. 2020;20(40):194354.

20. Sulyman D, Ayanda K, Dattijo L, Aminu B. Postnatal depression and its associated factors among Northeastern Nigerian women. Trop Med Public Heal. 2016;9(3):184-90.

21. Desta M, Memiah P, Kassie B, Ketema DB, Amha H, Getaneh T, Sintayehu M. Postpartum depression and its association with intimate partner violence and inadequate social support in Ethiopia: a systematic review and meta-analysis. J Affect Disord. 2020;

22. Dadi AF, Akalu TY, Baraki AG, Wolde HF. Epidemiology of postnatal depression and its associated factors in Africa: A systematic review and meta-analysis. PLoS One. 2020;15(4):e0231940.

23. Tester-Jones M, O'Mahen H, Watkins E, Karl A. The impact of maternal characteristics, infant temperament and contextual factors on maternal responsiveness to infant. Infant Behav Dev. 2015;40:1-1.

24. Ye Z, Wang L, Yang T, Chen LZ, Wang T, Chen L et al. Gender of infant and risk of postpartum depression: a meta-analysis based on cohort and case-control studies. J Matern Neonatal Med. 2020;1-10.

25. Kızılırmak A, Calpbinici P, Tabakan G, Kartal B. Correlation between postpartum depression and spousal support and factors affecting postpartum depression. Health care for women international. 2020;14:14.

26. Dekel S, Ein-Dor T, Berman Z, Barsoumian IS, Agarwal S, Pitman RK. Delivery mode is associated with maternal mental health following childbirth. Arch Womens Ment Health. 2019;22(6):817-24 
27. Upadhyay AK, Singh A, Singh A. Association between unintended births and risk of postpartum depression: Evidence from Ethiopia, India, Peru and Vietnam. SSM-population Heal. 2019;9:100495.

28. Underwood L, Waldie KE, D'Souza S, Peterson ER, Morton SM. A longitudinal study of pre-pregnancy and pregnancy risk factors associated with antenatal and postnatal symptoms of depression: evidence from growing up in New Zealand. Matern Child Health J. 2017;21(4):915-31.

29. Azad R, Fahmi R, Shrestha S, Joshi H, Hasan M, Khan AN et al. Prevalence and risk factors of postpartum depression within one year after birth in urban slums of Dhaka, Bangladesh. PLoS One. 2019;14(5):e0215735.

30. Wittkowski A, Gardner PL, Bunton P, Edge D. Culturally determined risk factors for postnatal depression in Sub-Saharan Africa: A mixed method systematic review. J Affect Disord. 2014;165:115-24.

31. Chee CY, Lee DT, Chong YS, Tan LK, Ng TP, Fones CS. Confinement and other psychosocial factors in perinatal depression: A transcultural study in Singapore. J Affect Disord. 2005;89(1-3):157-66.

32. Atuhaire C, Brennaman L, Cumber SN, Rukundo GZ, Nambozi G. The magnitude of postpartum depression among mothers in Africa: a literature review. Pan Afr Med J. 2020;37.

33. Smorti M, Ponti L, Pancetti F. A comprehensive analysis of postpartum depression risk factors: the role of sociodemographic, individual, relational, and delivery characteristics. Front public Heal. 2019;7:295.

34. Kariuki EW, Kuria MW, Were FN, Ndetei DM. Effectiveness of a brief psychoeducational intervention on postnatal depression in the slums, Nairobi: a longitudinal study. Arch Womens Ment Health. 2021;24(3):503-11.

35. Kojima M, Furukawa TA, Takahashi H, Kawai M, Nagaya T, Tokudome S. Cross-cultural validation of the Beck Depression Inventory-II in Japan. Psychiatry Res. 2002;110(3):291-9.

36. Ghassemzadeh H, Mojtabai R, Karamghadiri N, Ebrahimkhani N. Psychometric properties of a persianlanguage version of the Beck Depression Inventory- second edition: BDI-II Persian. Depress Anxiety. 2005;21(4):185-92.

37. Ndetei DM, Khasakhala LI, Kuria MW, Mutiso VN, Ongecha-Owuor FA, Kokonya DA. The prevalence of mental disorders in adults in different level general medical facilities in Kenya: a cross-sectional study. Ann Gen Psychiatry. 2009;8(1):1-8.

38. Musyimi CW, Mutiso V, Nayak SS, Ndetei DM, Henderson DC, Bunders J. Quality of life of depressed and suicidal patients seeking services from traditional and faith healers in rural Kenya. Health Qual Life Outcomes. 2017;15(95):1-10.

39. Mokwena K, Masike. The Need for Universal Screening for Postnatal Depression in South Africa: Confirmation from a Sub-District in Pretoria, South Africa. Int J Environ Res Public Heal. 2020;17(19):6980.

40. Omole OB, Phukuta NS. Prevalence and risk factors associated with postnatal depression in a South African primary care facility. African J Prim Heal Care Fam Med. 2020;12(1):1-6.

41. Shorey S, Chee CY, Ng ED, Chan YH, San Tam WW, Chong YS. Prevalence and incidence of postpartum depression among healthy mothers: a systematic review and meta-analysis. J Psychiatr Res. 2018;104:235-48. 
42. Abebe A, Tesfaw G, Mulat H, Hibdye G. Postpartum depression and associated factors among mothers in Bahir Dar Town, Northwest Ethiopia. Ann Gen Psychiatry. 2019;18(1):1-8.

43. Jayaweera RT, Ngui FM, Hall KS, Gerdts C. Women's experiences with unplanned pregnancy and abortion in Kenya: A qualitative study. PLoS One. 2018;13(1):e0191412.

44. Hall KS, Dalton VK, Zochowski M, Johnson TR, Harris LH. Stressful life events around the time of unplanned pregnancy and women's health: exploratory findings from a national sample. Matern Child Health J. 2017;21(6):1336-48.

45. Barton K, Redshaw M, Quigley MA, Carson C. Unplanned pregnancy and subsequent psychological distress in partnered women: a cross-sectional study of the role of relationship quality and wider social support. BMC Pregnancy Childbirth. 2017;17(1):1-9.

46. Kim Y, Dee V. Sociodemographic and Obstetric Factors Related to Symptoms of Postpartum Depression in Hispanic Women in Rural California. J Obstet Gynecol Neonatal Nurs. 2018;47(1):23-31.

47. Dlamini LP, Mahanya S, Dlamini SD, Shongwe MC. Prevalence and factors associated with postpartum depression at a primary healthcare facility in Eswatini. South African J Psychiatry. 2019;25(1):1-7.

48. Eastwood JG, Phung H, Barnett B. Postnatal depression and sociodemographic risk: factors associated with Edinburgh Depression Scale scores in a metropolitan area of New South Wales. Aust New Zeal J Psychiatry. 2011;45(12):1040-6.

49. Faramarzi M, Esmailzadeh S, Mosavi S. A comparison of abused and non-abused women's definitions of domestic violence and attitudes to acceptance of male dominance. Eur J Obstet Gynecol Reprod Biol. 2005;122(2):225-31.

50. Ogbo FA, Eastwood J, Hendry A, Jalaludin B, Agho KE, Barnett B, Page A. Determinants of antenatal depression and postnatal depression in Australia. BMC Psychiatry. 2018;18(1):49.

51. Giallo R, Gartland D, Woolhouse H, Brown S. "I didn't know it was possible to feel that tired": exploring the complex bidirectional associations between maternal depressive symptoms and fatigue in a prospective pregnancy cohort study. Arch Womens Ment Health. 2016;19(1):25-34.

52. Giallo R, Gartland D, Woolhouse H, Brown S. Differentiating maternal fatigue and depressive symptoms at six months and four years post partum: Considerations for assessment, diagnosis and intervention. Midwifery. 2015;31(2):16-22.

\section{Figures}




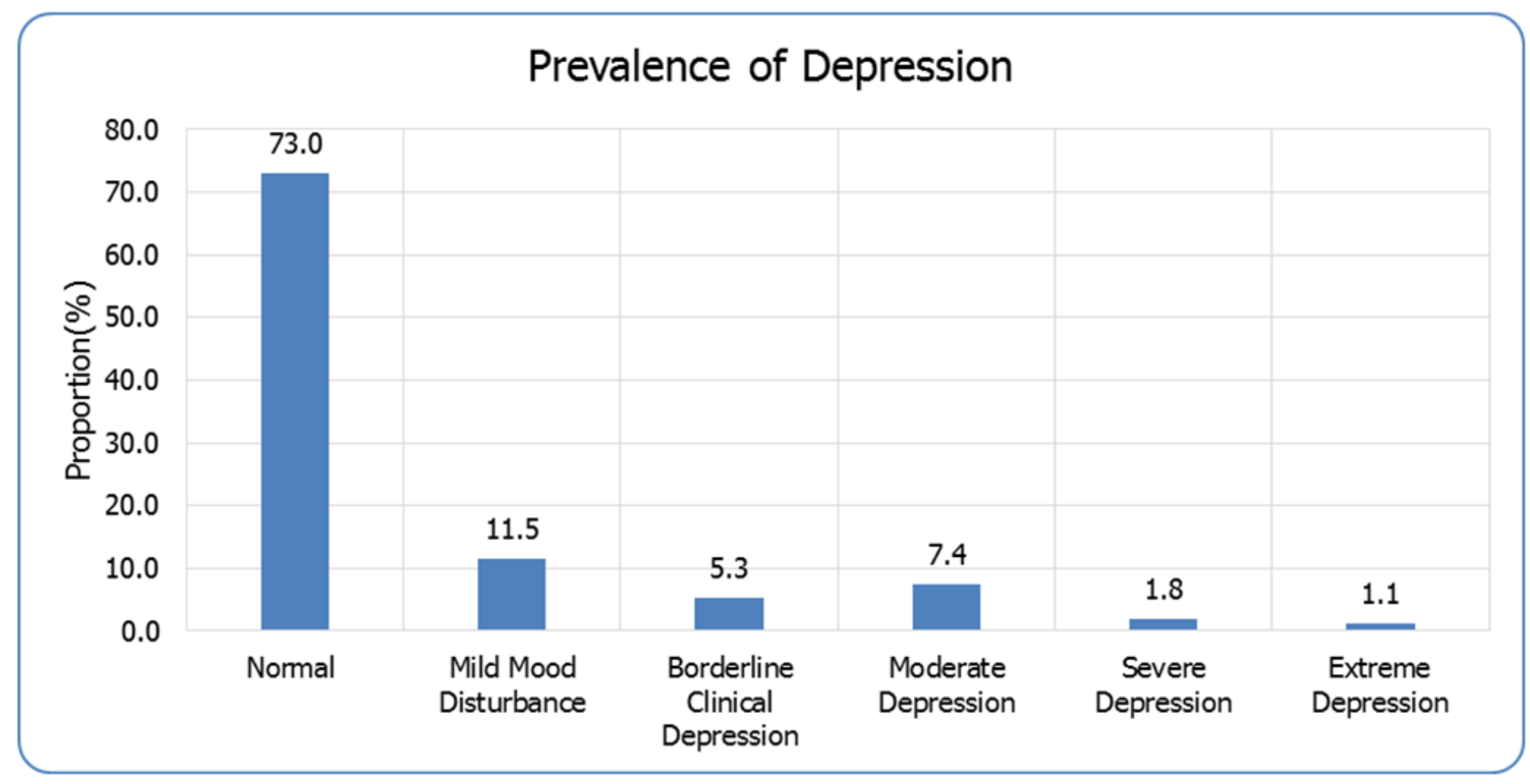

Figure 1

Prevalence of depression 